\title{
Recent Advances in Clay/Polymer Nanocomposites
}

\author{
N. Bitinis, M. Hernandez, R. Verdejo, J. M. Kenny, M. A. Lopez-Manchado* \\ Instituto de Ciencia y Tecnología de Polímeros, ICTP-CSIC, Juan de la Cierva, 3 28006- \\ Madrid (Spain) \\ *Corresponding author: Imanchado@ictp.csic.es
}

Composite materials represent one of the most active fields in the polymer industry. Many different types of fillers, carbon black, calcium carbonate, glass fibres and talc in the micrometer size range have been added to polymers to provide an improvement of the final product properties. However, this improvement is only achieved at high filler concentrations, which lead to an increase in the viscosity of the material and, hence, problems in processing. In recent years, it has been observed that the addition of just a small quantity of nano-sized layered silicates greatly improved the properties of virgin polymers without affecting their processability. Layered silicate clays have been found to be effective reinforcing fillers for polymers due to their lamellar structure and high aspect ratio of about $750 \mathrm{~m}^{2} / \mathrm{g}$. Smectite clays (e.g. montmorillonite), belonging to the structural family called 2:1 phyllosilicates, are the main choice for designing polymer nanocomposites due to their low cost and rich intercalation chemistry allowing them to be chemically modified (organoclays) and to improve the compatibility with the polymer matrix. These hybrid materials, normally called polymeric nanocomposites (PNC), represent a radical alternative to conventional polymer composites and have focused the attention of both academia and industry because of their unexpected properties, and their straightforward synthesis and processing. ${ }^{[1]}$ The ideal design of a nanocomposite involves individual silicate layers (1 nm thick) homogeneously dispersed 
in a continuous polymer. The uniform dispersion of nanoelements can lead to an ultra-large interfacial area between the constituents per volume of material. Thus, in PNCs with only a low volume percentage of dispersed nanoparticles, the entire polymer matrix may be considered to be a nanoscopically confined interfacial polymer. Such materials on the nanoscale level show significant improvements in mechanical properties, heat distortion temperatures, thermal stability, flame retardancy and enhanced barrier properties. The combination of enhanced properties, weight reduction, and low cost has led to interesting commercial applications such as automotive and packaging, among others. All this justifies the growing interest of both academia and industry in the development of these hybrid materials.

We will now describe the most significant findings in the clay/polymer nanocomposites field considering three polymer families: elastomers, thermosets and from natural resources or biopolymers.

\section{Elastomer nanocomposites}

Rubbers, or most specifically called elastomers, are one of the most important commercial polymers. They are formed by long chains with molecular weights of the order of $1 * 10^{5} \mathrm{~g} / \mathrm{mol}$ crosslinked at many points along their length, producing 10-20 crosslinks per primary molecule, giving rise to the formation of a three-dimensional network structure. Their main characteristic is the ability to suffer considerable deformation under stress but upon release of the stress, the rubber article will go back to its original shape, recovering the energy stored during the deformation. 
The elastomers are soft and weak materials with a low modulus and strength, so they usually require the inclusion of fillers in order to get a substantial improvement in the physical and mechanical properties of the compound. A wide range of fillers have traditionally been used in the rubber industry, being carbon black and silica the main fillers used in compounding recipes. However, a minimum of $20-30 \mathrm{wt} \%$ of conventional fillers is needed to increase the properties, but, this high concentration may reduce the processability of the compound and increase the weight of the final material, limiting their applications. In this sense, the nano concept is highly relevant for rubber compounds since their application requires filler reinforcement. Several benefits in organoclay/rubber nanocomposites have been identified including mechanical properties, barrier properties, flame resistance and the wear behavior. $^{[2]}$ Thus, rubber nanocomposites with layered silicates have received increasing attention in recent years. Intercalated and partially exfoliated structures were successfully prepared by several processing techniques, such as melt compounding, solution intercalation, and latex-compounding.

Lopez-Manchado et al. prepared for the first time organoclay/natural rubber (NR) nanocomposites with a fully exfoliated structure via the vulcanization process. ${ }^{[3]}$ The authors observed that the cure characteristics of NR were affected by the organoclay. The addition of the nanofillers led to an increase in the vulcanization rate and to a sensible raise in the torque value as compared to neat NR, which indicates that a higher number of crosslinks were formed. This statement was supported by swelling measurements and differential scanning calorimeter isothermal studies, where an increase of the heat involved during the curing reaction in the presence of the organoclay was observed. Moreover, the organoclay behaved as an effective reinforcing agent for natural rubber, showing increments of more than $250 \%$ in 
the stress at several deformations. When comparing at similar filler contents with carbon black, the filler par excellence in the rubber world, it was seen that organoclay had a stronger reinforcing effect than carbon black. So only $10 \mathrm{phr}$ (parts per hundred of rubber) of organoclay are enough to obtain a similar mechanical behaviour as the compound with $40 \mathrm{phr}$ of carbon black. In addition, high contents of carbon black led to a marked loss in the elastic behaviour of the compound, while the organoclay retained the elasticity of the material. A similar behaviour for nitrile butadiene rubber (NBR) was observed by Okada et al. ${ }^{[4]}$ They found that the mechanical properties of NBR with $10 \mathrm{phr}$ were comparable to the compound with $40 \mathrm{phr}$ carbon black. According to these results, the substitution of carbon black by organoclay may be straightforward as the overall filler content in the compound can be highly reduced. The improved properties of these nanocomposites are usually attributed to the formation of an intercalated/exfoliated structure, in which enough polymer chains are inserted into the silicate galleries, so the polymer is nanoscopically confined. It is evident, that an optimum dispersion of the filler into the polymer matrix gives rise to a strong filler/polymer interfacial adhesion, however this account is not sufficient to paint a complete picture of the reinforcing mechanism taking place in many systems. Hence, it is critical to carry out basic research to get a more thorough understanding of the nanofiller/polymer interaction mechanisms. In the particular case of rubber compounds, it is important to know the effect of these nanoparticles on the network structure. Towards this aim, Lopez-Manchado et al. ${ }^{[5,6,7]}$ evaluated the influence of inorganic nanoparticles on crosslinking mechanism of elastomers by means of a theoretical approach ${ }^{[5]}$, in-situ SAXS studies ${ }^{[6]}$ and molecular dynamics analysis by dielectric spectroscopy. ${ }^{[7]}$ The authors first applied the tube model ${ }^{[5]}$ on equilibrium statistical mechanics of elastomers, which is one of the most challenging 
problems in polymer physics. This model allows a proper separation of the contributions from the chemical crosslinks and constraints to the mechanical behaviour. The authors observed that both fillers increased the contribution corresponding to crosslinks, enhancing the network density, due to the formation of a higher number of interactions. This effect was more evident in the presence of organoclay due to a deep interaction filler/matrix at the interphase. In addition, the average molecular mass of the network chains, $M c$, decreased by the addition of both fillers, in particular, when the organoclay is used as nanoreinforcement. The authors deduced a different filler/elastomer interaction mechanism for both fillers. Carbon black has reactive groups on its surface likely to form strong covalent bonds with the elastomer, limiting the movement of the chains, so, the dimensions of the tube are reduced, but this interaction is restricted to a zone at the filler/elastomer interface, which is only a small rubber percentage. The rest of the rubber chains present freedom for mobility. However, the nanoparticles due to its inorganic nature mainly exhibit physical absorptions with the elastomer. So, in a particular interphase, the interaction with the elastomer is weaker than carbon black composite, and then, the mobility of the chains is higher. However, these nanofillers are characterised by a high aspect ratio; therefore, the optimum dispersion of even small amount of nanoparticles is enough to interact with the total rubber mass. This results in the formation of a highly ordered structure due to the formation of intense nanoparticle/elastomer interactions, in which, the mobility of rubber chains is surrounded in its lateral fluctuations by the presence of neighbouring chains. Hence, the molecular orientation of the chains is higher, and, further additional crosslinks in the system are formed. By observing the stress-strain curves, it was deduced that at low deformations, the elastic response of the material is given by both contributions (crosslinks and entanglements). These 
entanglements have a high effectiveness at low elongations since present a limited fluctuation between two crosslinks points. As the material is stretching, the physical links slippage, losing effectiveness, which explains the decrease of the strength. This effect is more evident in the presence of organoclay. At high strains, when the slippage of the constraints is hindered by the chemical crosslinks, these physical links act as additional crosslinks in the network structure, increasing the strength. This effect is very important to explain the final properties of the material, since it increases the toughness of the compound. To corroborate the molecular origin of the theoretical approach, the authors studied the induced crystallisation phenomenon of natural rubber under stretching. Carretero et al ${ }^{[6]}$ demonstrated that the organoclay promoted the strain-induced crystallisation in natural rubber nanocomposites. The addition of nanoclay led to significantly enhanced crystallinity and lower onset strain for stretch-induced crystallisation compared to conventionally filled NR composites. Crystallisation of neat NR seems to occur in a single step once a critical value of strain $\alpha=$ 3.4 is reached. In contrast, crystallisation in the nanocomposites started at $\alpha=1.2$ and appeared to follow a more complex, two-step pattern (Figure 1). Although the $I_{200}$ intensity values are directly related to the amount of NR crystallites formed during stretching, it must be taken into account that the motion of the nanoclay particles and the motion of the polymer chains must be coupled. The first step for $\alpha$-values $<3$ is most likely related to the orientation and alignment of the highly anisotropic nanoclay particles. In a second step $(3 \leq \alpha \leq 4)$, these highly anisotropic nanoparticles can be completely aligned along the direction of the deformation and, thus a physical network is formed. This physical network may favour the alignment of the rubber chains and the crystallisation rate could increase. The final study was carried out by Hernandez et al. ${ }^{[7]}$ and evaluated the effect of the organoclay on the molecular 
dynamic of polymer nanocomposites by dielectric spectroscopy. A new relaxation ascribed to a restricted segmental mode appeared in the NR/organoclay nanocomposites regardless of the vulcanisation of the NR matrix. This "new mode" was attributed to the segmental dynamics of polymer chains at the interfacial polymer particle regions. NR exhibits two relaxation modes, a segmental motion (segmental mode) at temperatures above the glass transition temperature $\left(T_{g}=-64{ }^{\circ} \mathrm{C}\right)$ associated to the segmental motions of the polymer chain, and a slower one related to the relaxation of whole chain (normal mode). The normal mode disappears after crosslinking due to suppression of large-scale motions of the dipoles oriented parallel to the polymer backbone. Vulcanised NR exhibits an intense relaxation around 237.5 $\mathrm{K}$, associated with the segmental mode (Figure 2). No significant changes in the relaxation behaviour were observed when the nanoclay was added. This indicates that the segmental motion of cis-polyisoprene is not disturbed by the addition of the nanoparticles. As observed in the figure, organoclay/NR nanocomposites show a slower relaxation at high frequencies associated with a Maxwell-Wagner-Sillars (MWS) process induced by the accumulation of charges at the polymer-nanoclay interfaces. In addition, the presence of a second peak at high frequencies associated with a new mode is observed in the nanocomposites. This new mode is slower than the segmental one with a new effective $T_{g}$ of $90 \mathrm{~K}$ higher than neat polymer. It can be associated to a restricted segmental dynamic due to cooperative movements of the polymer chains in the interface layer around the clay particles. Both segmental and new modes exhibit equivalent shifts in their temperature with vulcanisation. Thus, this fact further supports the assumption that the "new mode" of the NR-organoclay compounds corresponds effectively to a restricted segmental relaxation. The authors considered that part of the 
polymer chains are partially immobilised as an interface layer around the clay particles, and so the interfacial effects dominate the bulk properties of the material.

After this thorough research, the authors concluded that the inclusion of highly anisotropic nanoparticles leads to microscale segmental variations, giving rise to the formation of a highly ordered structure due to the formation of intense nanoparticle/elastomer interactions. The presence of these interfacial adhesions induces an early promotion and overall enhancement of the crystallization of NR chains under uniaxial stretching. The interplay between all these parameters could explain why the reinforcing effects in clay/natural rubber nanocomposites are manifested at low filler loadings.

\section{Thermosetting matrices nanocomposites}

Epoxy matrices, for their wide utilization on advanced composites for aeronautical, transport and electronic applications have been certainly the most studied thermosetting polymers for new nanocomposites with clay nanoplatelets. The potential of the use of smectite clays in the production of epoxy matrix nanocomposites has been early reported by Pinnavia ${ }^{[8]}$ who later deeply analyzed the mechanisms for the intercalation and exfoliation of compatibilized clay nanoplatelets by the most commonly used epoxy systems ${ }^{[9]}$. Later, Berglund et al. ${ }^{[10]}$ demonstrated the influence of the cation-exchange capacities of different clays on the intercalation and exfoliation efficiency in the preparation of epoxy matrix nanocomposites

Certainly, the preparation of well intercalated and exfoliated epoxy matrix clay nanocomposites has been already assessed and extensively reported. Nowadays, most of the research efforts are focused on the achievement of complex hybrid systems with multifunctional properties for advanced applications. 
Probably, the thermal stability and fire resistance has been one of the main properties where research is still focused after the classic paper published by Gilman, Kenny et al. ${ }^{[12]}$ observed that the addition of montmorillonite to a DGEBA monomer cured with anhydrides showed a clear catalytic effect and an increase of the thermal stability of the nanocomposite in presence of oxygen compared to the neat resin. Such result prompted the study of the possible synergic effects of organoclays and classical flame retardants, such as phosphorous based flame retardant, resulting on an evident catalytic action on the cure reactions. This improved flame retardancy has also been observed in thermoplastic matrix clay nanocomposites. In particular, Cardenas et al. ${ }^{[13]}$ reported the positive effects of the addition of organo-modified synthesized bentonite on the thermal stability and flame resistance of EVA, while Dasari et al. ${ }^{[14]}$ reported significant reductions on the heat released of organoclay reinforced polyamide 6 .

The development of novel processing routes for the development of well dispersed clay/epoxy nanocomposites is also an active current topic of research. In this respect Kenny et al. ${ }^{[15]}$ analysed the synthesis of these materials by the Frontal Polymerisation (FP) process. FP exploits the exothermicity of the self-same polymerisation reaction. It the heat dissipated is not excessive, the quantity of energy left over may be sufficient to induce the polymerisation of the monomer layer close to the zone heated by the reaction. As a result, a hot propagating and self-sustaining front is formed. FP runs are generally characterised by a constant velocity of the front and a very uniform temperature profile leading to clear advantages in terms of reaction time, uniformity of curing conditions and energy savings. The obtained nanocomposites showed characteristics similar or even better than those obtained by conventional polymerisation routes. However, the easy synthesis protocol and the extremely 
reduced preparation times demonstrated the applicability and advantages of this process to manufacture clay/epoxy nanocomposites.

The influence of clay addition has been also analysed in the case of phenolic resins ${ }^{[16]}$. It was found that the addition of clays with modifiers containing hydrophilic groups led to less crosslinked polymer networks than in the case of the resol, because of the interaction between the modifier and the prepolymer. However, a strong intercalation of the resol into the montmorillonite galleries and subsequent exfoliation lead to an increase of the hardness and elastic modulus of the nanocomposite.

The catalytic effects of nanoclays in thermosetting resins have promoted different studies on the curing kinetics and on the development and application of existing and new kinetic models for thermosetting matrix nanocomposites. A complete chemorheological analysis has been recently applied to epoxy/amine/montmorillonite nanocomposites combining a thermokinetic analysis of the curing reaction and chemorheological characterisation. ${ }^{[17]}$ The comparison of DSC thermograms has shown that although the addition the nanofiller did not change the mechanism of crosslinking, the nanoclay platelets produce an evident hindrance on the molecular mobility, which in turn influences the cure reactions. As none of the kinetic models available in literature was able to describe the cure behaviour of the aforementioned materials, a new phenomenological model was proposed considering the changes of the activation energy of the crosslinking process as a function of the degree of cure. Regarding the chemorheology, the viscosity of the nanocomposite was higher than the pure resin at any temperature; furthermore gelation occurred at lower degrees of cure, thus determining a narrow processing window. The applied chemorheological model was a modified version of the classical Williams-Landel-Ferry (WLF) equation. 


\section{Bionanocomposites}

Bionanocomposites are a promising class of hybrid nanostructured materials formed by the combination of polymers coming from natural resources (biopolymers) or synthetic biofunctional polymers and inorganic/organic solids at nanometric scale. They couple the intrinsic properties of natural polymers, biocompatibility and biodegradability, with the typical properties of nanocomposites derived from synthetic polymers, such as improved mechanical properties, higher thermal stability, and enhanced barrier properties. These novel environmental-friendly materials open new scenarios for biodegradable polymers with potential perspectives for medicine, coatings, automotive, packaging applications, etc.

Today, there is an urgent need for the development of environmentally friendly or "green" polymeric materials with the aim of replacing petroleum-derived synthetic plastics. It has been widely stated that the addition of inorganic fillers increase the mechanical properties, thermal stability and barrier properties of the bioplastics. ${ }^{[18]}$ Another interesting and exciting behaviour of biodegradable polymers-based nanocomposites is the significant improvement of the biodegradability of bioplastics. It has been demonstrated that the biodegradability of neat biopolymers is strongly accelerated by addition of organoclay likely due to a catalytic role of the nanofiller. ${ }^{[19]}$ All these features make bionanocomposites a promising material for the production of recyclable eco-friendly packaging materials based on renewable resources that help to reduce the environmental impact of plastic waste.

Other applications of bionanocomposites are related to electrochemical devices and biomedical applications, due to its biodegradable, non-toxic and easy processing. Ruiz-Hitzky et al. reported for the first time a new class of hybrid materials based on $\mathrm{Na}^{+}-$ 
montmorillonite/chitosan bionanocomposites exhibiting anion-exchange ability suitable to build potentiometric sensors. ${ }^{[20]}$ The intercalation of chitosan in layered silicates appears as an improved way to develop robust, stable and handy three-dimensional nanocomposites, that can be combined with graphite to construct bulk-modified electrodes useful for anionic detection in aqueous media. Chitosan is a polysaccharide derived from chitin, one of the main constituents of the exoskeleton of crustaceans and some insects. Positively charged chitosan chains are easily intercalated in the interlayer space of montmorillonite by an ion-exchange mechanism. In acidic solutions, chitosan shows an extended structure that may facilitate the biopolymer intercalation in the clay interlayer space in opposition to analogous polysaccharides with coiled or helicoidal structures that are only adsorbed on the external surface of clays. Chitosan chains are absorbed on the silicate with a mono or bilayer configuration as a function of chitosan concentration added to the clay suspension (Figure 3). The intercalation of the first layer of chitosan takes place mainly by electrostatic interactions between the $-\mathrm{NH}_{3}^{+}$groups in the chitosan chain and the negative sites in the clay. In contrast, hydrogen bonds between amino and hydroxyl groups of chitosan and the clay substrate are established in the adsorption of the second layer. The excess protonated amino groups that do not interact electrostatically with the clay layer remain available as anion-exchange sites and, in this way, such nanocomposites become suitable systems for the detection of anions showing a higher selectivity toward monovalent anions. These materials prepared as selfsupporting films show a potential interest as membranes for different processes related to separation of ions and gases, as well as components in electrochemical devices (fuel-cell, potentiometric sensors). 
Ruiz-Hitzky et al. ${ }^{[21]}$ also developed potentiometric sensors based on layered double hydroxides (LDH) and negatively charged polysaccharides such as alginic acid, pectin, kcarrageenan, and xanthan gum by co-precipitation or co-organized assembly mechanism. In this case, the anionic exchange ability of the pristine $\mathrm{LDH}$ was turned into a cationic exchange capacity due to negatively charged groups in the polysaccharide structure that do not interact with the positively charged LDH layers. These devices can be applied to the determination of calcium ions and other divalent cations.

Also, they developed a novel multifunctional material derived from cheap, abundant and environmental friendly products, such as table sugar (sucrose) and bentonite clays (layer silicates) with excellent properties as electrode materials. ${ }^{[22]}$ This nanomaterial which was denoted as "caramel/clay nanocomposite" was formed when a mixture of clay and sucrose was heated by microwave (MW) irradiation. This type of irradiation activates the reactions, reducing the reaction time and leading to the formation of a reproducible caramel/clay nanocomposite following an intercalative caramelisation process. These caramel/clay nanocomposites are excellent precursors of carbon/clay nanocomposites. The caramel is converted almost entirely into carbonaceous material, when the nanocomposite is heated at $750{ }^{\circ} \mathrm{C}$ under $\mathrm{N}_{2}$ flow during $3 \mathrm{~h}$, as was confirmed by thermogravimetric analysis and ${ }^{13} \mathrm{C}$ NMR. In addition, the thermal treatment of the nanocomposite led to a marked increase, almost by a factor of 50, of the specific surface area of the material due to the nanostructured arrangement of the components (silicate and intercalated caramel) or to the catalytic activity of the clay in the carbonisation process. The carbonization process was followed by in-situ electrochemical impedance spectroscopy (EIS), where it was observed that the formation of carbon strongly increased the electrical conductivity reaching values close to $10^{-1} \mathrm{Scm}^{-1}$. This 
conductivity was slightly reduced after cooling the resulting material giving values close to $10^{-4} \mathrm{Scm}^{-1}$ at room temperature. The conductivity values of these carbon/clay nanocomposites together with their relatively high specific surface make these low cost materials suitable to prepare electroactive components for electrochemical devices, such as lithium rechargeable batteries, sensors, and biosensors, supercapacitators and electrocatalysts. Furthermore, the authors functionalised the material by an organosilinisation reaction, incorporating desired functional groups, for example protonated amino groups, to act as anion-exchange sites. Thus, the nanocomposite can simultaneously operate as electronic collector and sensing phase in a potentiometric sensor. In addition, the material showed a reduced porosity that stopped anions of large volume, making the sensor specific to small monovalent anions.

Another important application of the bionanocomposites is in the biomedical field. ${ }^{[23]}$ Wang et al. successfully prepared a drug delivery system based on quaternized chitosan/montmorillonite nanocomposites by melt mixing. ${ }^{[23 a]}$ The authors observed that the montmorillonite enhanced the drug encapsulation efficiency and slowed the drug release of the chitosan. The intercalation of the biopolymer chains into the silicate clays led to a synergy, combining the drug absorption action and the mucosa protection effect of the clay with the mucoadhesive and permeability of the biopolymer. In addition, the nanocomposites so obtained were non-citotoxic, which makes them a material of the great potential for biomedical applications.

Bionanocomposites were also tested for a gene delivery system. Wang et al. developed a novel and potential non-viral gene carrier based on a hybrid material of quaternized chitosan intercalated into the silicate layers of a rectorite. ${ }^{[23 \mathrm{~b}]}$ The toxicity studies revealed that the bionanocomposites were biocompatible and non-toxic. In addition, in-vitro cell transfection 
studies indicated the ability of the bionanocomposites to absorb DNA through electrostatic interactions whereas in-vivo transfection study showed the higher DNA transfection of the bionanocomposites in relation to the pristine polymer.

Recently, Ruiz-Hitzky et al. developed a new hybrid material based on the combination of layered double hydroxide (LDHs) and two biopolymers (a protein, zein and a polysaccharide, alginate), able to act as effective drug delivery system. ${ }^{[23 c]}$ Ibuprofen was chosen as drug model being intercalated into the $\left[\mathrm{Mg}_{2} \mathrm{Al}\right] \mathrm{Cl} \mathrm{LDH}$ by an ion exchange reaction. In-vitro tests revealed that the bionanocomposite showed a better protection against drug release at the stomach $\mathrm{pH}$ and a controlled liberation in the intestinal tract conditions. The reported approach can be extended to the immobilisation of several drugs as well as to enlarge its application in topical therapies. The use of bionanocomposites as carriers for influenza was recently evaluated. ${ }^{[23 \mathrm{~d}]}$ Ruiz-Hitzky et al. developed a new bionanocomposite by assembly of a polysaccharide (xanthan gum) and a microfibrous silicate sepiolite. The negative charges in the nanocomposite electrostatically interacted with the positively charged molecules present at the surface of the viral particles. Experiments carried out in mice demonstrated that the biohybrid material increased the immune response against the influenza virus. Also, the material showed the ability to retain water, providing an environment favourable for biomolecules.

Zhuang et al. ${ }^{[23 \mathrm{e}]}$ developed an intercalated gelatin/montmorillonite-chitosan bionanocomposite with great potential in tissue engineering by a solution intercalation process. The nanocomposite had a lower degradation rate than the composite as confirmed by in-vitro degradation tests due to the protecting effect of the montmorillonite on the polymer against the action of lysozyme present in the body fluids. On the other hand, the cell adhesion 
studies provided evidences of a good adhesion, proliferation and morphology of rat stromal stem cells on nanocomposite membranes as compared to the tissue culture plates.

\section{Conclusions and Outlook}

The development and processing of nanoclay/polymer matrix nanocomposites certainly represent one the most dynamic research areas in polymer science and technology of the past two decades. Nanoclays have provided an accessible and low cost additive to enhance the properties of nanostructured polymeric materials for a wide selection of industrial applications and have opened, since the first reports by Toyota, the door for further developments of polymer nanotechnologies. The first efforts were devoted to analyse the effects of the addition of nanoclays in practically all the imaginable properties of any possible polymer matrix: improvement in mechanical, barrier, solvent resistance, thermal stability, flame retardance and many other properties have been extensively reported. Moreover, the versatility of nanoclays has been enhanced by the wide range of possible organo-modifiers able to promote the compatibilisation with different polymers leading to the desired intercalation and exfoliation morphologies.

The research opportunities offered by these materials have not ended after the huge number of literature reports on processing and properties of clay nanocomposites. Research efforts are now concentrated in the possibility of functionalising the nanoclays in order to develop materials with specific functional properties for applications in advanced fields. This strategy is now leading to breakthrough results in theranostics, drug release, sensors, functional coatings and other applications in biomedicine, electronics and energy sectors. 
The development of chemical routes for the synthesis of new nanoclays and the tailored modification of the nanoclay structure and surface will ensure further progress and new research opportunities on these materials.

\section{Acknowledgments}

Financial support provided by the Ministerio de Ciencia e Innovación (MCINN) through the Project MAT 2010-18749. N.B. thanks the CSIC for a JAE-pre contract.

\section{References}

[1] a) M. Alexandre, P. Dubois. Mater Sci Engn R. 2000, 28, 1. b) Polymer Nanocomposites: Synthesis, characterization, and modeling (Eds. R. Krishnamoorti, R.A. Vaia), American Chemical Society, Washington, DC 2002. c) Polymer Nanocomposites (Eds. Y.W. Mai, Z.Z. Yu), CRC Press, Boca Raton, USA, 2006. d) Processing and Properties of Nanocomposites (Eds. S.G. Advani), World Scientific Publishing Co, London 2007. e) Processing and Properties of Nanocomposites (Ed. S.G. Advani) World Scientific Publishing, Singapore 2007. f) Recent Advances in Polymer Nanocomposites (Eds. S. Thomas, G.E. Zaikov, S.V. Valsaraj), VSP, Leiden, 2009. g) Recent Advances in Polymer Nanocomposites (Eds. S. Thomas, G.E. Zaikov, S.V. Valsaraj), VSP, Leiden, 2010.

[2] a) M. Arroyo, R. Zitzumbo, F. Avalos, J.L. Valentin, M.A. Lopez-Manchado, Encyclopedia of Nanoscience and Nanotechnology (Eds. H.S. Nalwa), American Scientific Publishers, California, USA 2010, 13, 215. b) Rubber Nanocomposites: Preparation, 
Properties and Applications (Eds. S. Thomas, R. Stephen), John Wiley \& Sons, Singapore 2010.

[3] a) M. Arroyo, M.A. Lopez-Manchado, B. Herrero, Polymer 2003, 44, 2447. b) M.A. Lopez-Manchado, B. Herrero, M. Arroyo, Polym. Int. 2003, 52, 1070. c) M.A. LopezManchado, M. Arroyo, B. Herrero, J. Biagiotti, J. Appl. Polym. Sci. 2003, 1, 1 d) M.A. Lopez-Manchado, B. Herrero, M. Arroyo, Polym. Int. 2004, 53, 1766

[4] A. Okada, A. Usuki, T. Kurauchi, O. Kamigaito, in Hybrid Organic-Inorganic Composites (Eds. J.E. Mark, C.Y.C. Lee and P.A. Bianconi), ACS Symposium Series 585, ACS, Washington, D.C. 1995

[5] M.A Lopez-Manchado, J.L. Valentin, J. Carretero, F. Barroso, M. Arroyo, Eur. Polym. J. 2007, 43, 4143

[6] a) J. Carretero-Gonzalez, R. Verdejo, S. Toki, B.S. Hsiao, E.P. Giannelis, M.A. LopezManchado, Macromolecules. 2008, 41, 2295. b) J. Carretero-Gonzalez, H. Retsos, R. Verdejo, S. Toki, B.S. Hsiao, E.P. Giannelis, M.A. Lopez-Manchado, Macromolecules. 2008, 41, 6763.

[7] a) J. Carretero-Gonzalez, H. Retsos, E.P. Giannelis, T.A. Ezquerra, M. Hernandez, M.A. Lopez-Manchado, Soft Matter, 2009, 5, 3481. b) M. Hernandez, J. Carretero-Gonzalez, R. Verdejo, T.A. Ezquerra, M.A. Lopez-Manchado, Macromolecules, 2010, 43, 643.

[8] T.J.Pinnavaia, Science, 1983, 220, 365

[9] T. Lan, T.J. Pinnavaia, Chem. Mater., 1994, 6 (12), 2216

[10] X. Kornmann, H. Lindberg and L. A. Berglund, Polymer, 2001, 42 (4), 1303

[11] J.W. Gilman, Applied Clay Science, 1999, 15 (1-2), 31 
[12] (a) L. Torre, E. Frulloni, J.M. Kenny, C. Manferti, G. Camino. J. Appl. Polym. Sci. 2003, 90, 2532. b) L. Torre, G. Lelli, J.M. Kenny. J. Appl. Polym. Sci. 2004, 94, 1676.

[13] M.A. Cardenas, D. Garcia-Lopez, J.F. Fernandez, I. Gobernado-Mitre, J.C. Merino, J.M. Pastor, J. de Dios Martinez, J. Barbeta, D. Calveras. Macromol. Mater. Eng. 2007, 292, 1035. [14] A. Dasari, Z.Z. Yu, Y.W. Mai, G. Cai, H. Song, Polymer. 2009, 50, 1577.

[15] A. Mariani, S. Bidali, G. Caria, O. Monticelli, S. Russo, J.M. Kenny. J. Appl. Polym. Sci. 2007, 45, 2204.

[16] a) L.B. Manfredi, D. Puglia, J.M. Kenny, A. Vazquez. J. Appl. Polym. Sci. 2007, 104, 3082. b) L.B. Manfredi, D. Puglia, A. Tomasucci, J.M. Kenny, A. Vazquez. Macromol. Mater. Eng. 2008, 293, 878.

[17] G. Lelli, A. Terenzi, J.M. Kenny, L. Torre. Polym. Compos. 2009, 30, 1

[18] a) S.S. Ray, M. Bousmina. Prog. Mater. Sci. 2005, 50, 962. b) J.K. Pandey, A.P. Kumar, M. Misra, A.K. Mohanty, L.T. Drzal, R.P. Singh. J. Nanosci. Nanotechnol. 2005, 5, 497. c) L. Yu, K. Dean, L. Li. Prog. Polym. Sci. 2006, 31, 576.

[19] a) J.A. Tetto, D.M. Steeves, E.A. Welsh, B.E. Powell. ANTEC'99 1628. b) S.R. Lee, H.M. Park, H.L. Lim, T. Kang, X. Li, W.J. Cho, C.S. Ha, Polymer. 2002, 43, 2495. c) S. Sinha Ray, K. Yamada, M. Okamoto, K. Ueda. Polymer. 2003, 44, 857

[20] a) M. Darder, M. Colilla, E. Ruiz-Hitzky. Chem. Mater. 2003, 15, 3774. b) E. RuizHitzky, M. Darder, P. Aranda. J. Mat. Chem. 2005, 15, 3650. c) M. Darder, M. Lopez-Blanco, P. Aranda, A.J. Aznar, J. Bravo, E. Ruiz-Hitzky. Chem. Mater. 2006, 18, 1602. d) M. Darder, P. Aranda, E. Ruiz-Hitzky. Adv. Mater. 2007, 19, 1309. e) E. Ruiz-Hitzki, M. Darder, P. Aranda, K. Ariga. Adv. Mater. 2010, 22, 323. f) E. Ruiz-Hitzky, P. Aranda, M. Darder, G. Rytwo. J. Mater. Chem. 2010, 20, 9306. 
[21] M. Darder, M. Lopez-Blanco, P. Aranda, F. Leroux, E. Ruiz-Hitzky. J. Mater. Chem. 2005, 17, 1969.

[22] M. Darder, E. Ruiz-Hitzky. J. Mat. Chem. 2005, 15, 3913.

[23] a) X.Y. Wang, Y.M. Du, J.W. Luo. Nanotechnology. 2008, 19, 065707. b) X.Y. Wang, X.F. Pei, Y.M. Du, Y. Li. Nanotechnology. 2008, 19, 375102. c) A.C.S. Alcantara, P. Aranda, M. Darder, E. Ruiz-Hitzky. J. Mater. Chem. 2010, 20, 9495. d) E. Ruiz-Hitzky, M. Darder, P. Aranda, M.A. Martin, G. del Real. Adv. Mater. 2009, 21, 4167. e) H. Zhuang, J.P. Zheng, H. Gao, K.D. Yao. J. Mater. Sci.-Mater. M. 2007, 18, 951. 
(a)
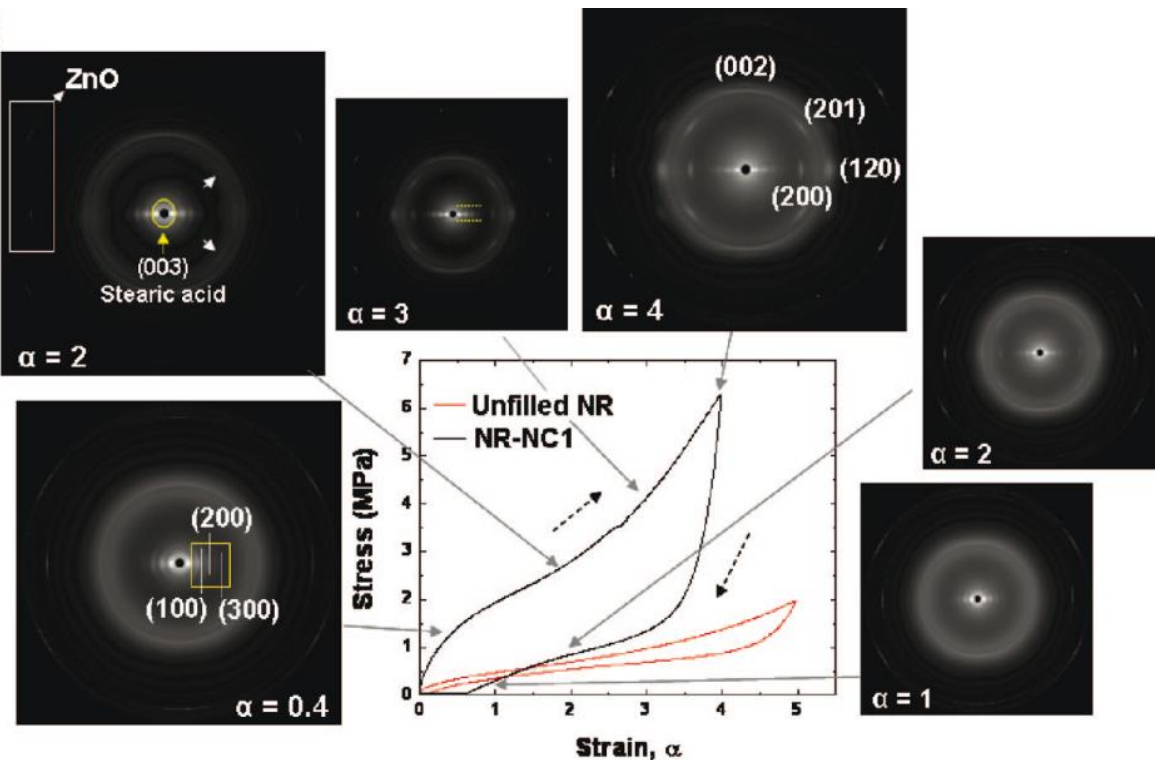

(b)

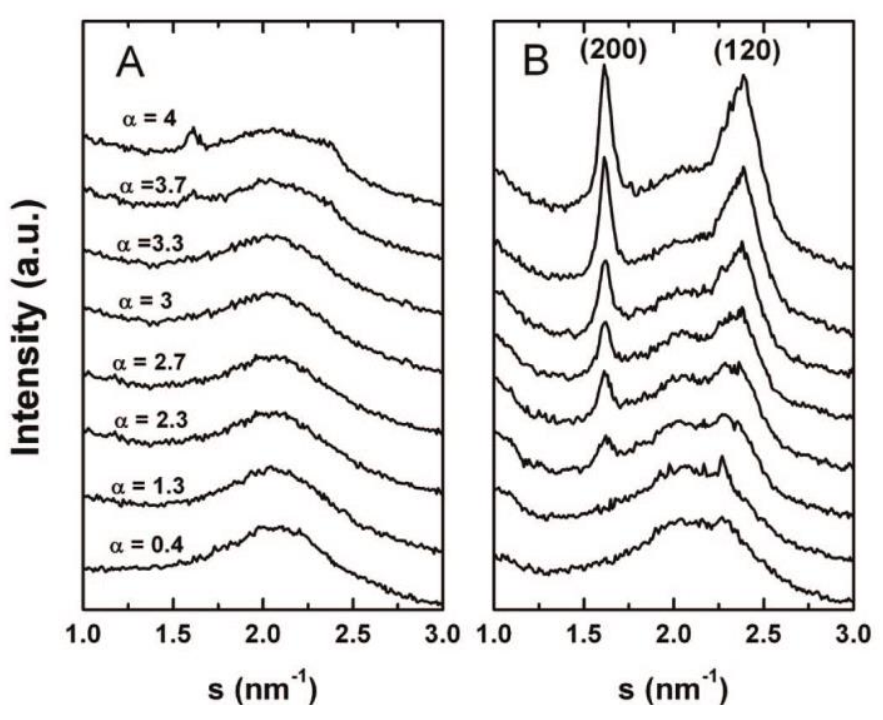

Figure 1. WAXD results for organoclay/NR nanocomposites. (a) Stress-strain curve and selected synchrotron WAXD patterns during extension and retraction cycles. (b) Integrated and corrected intensity profiles from the 2D WAXD patterns at various strains as a function of scattering vector s (nm-1) Reproduced from ref. [6a] with permission from American Chemical Society. 

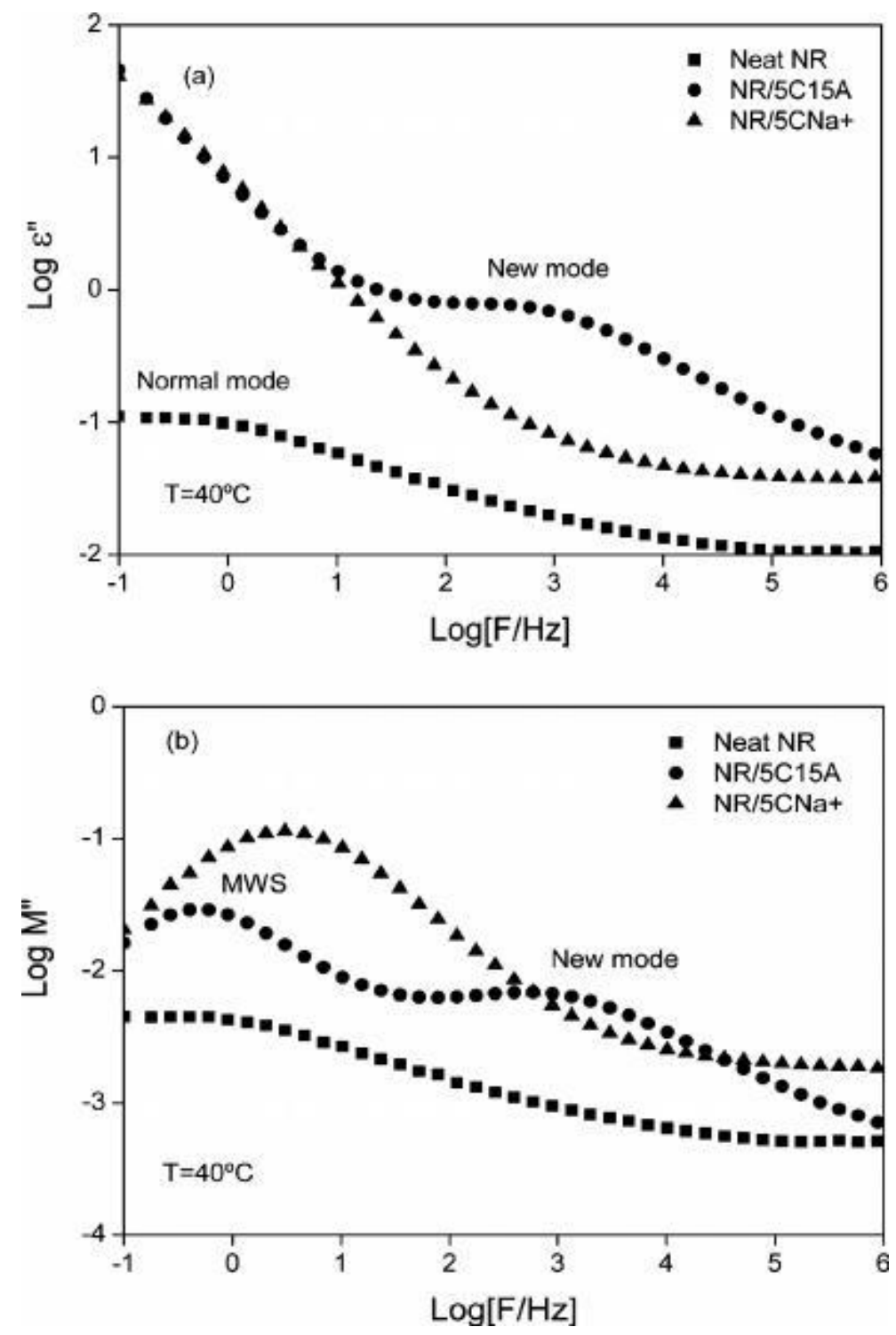

Figure 2. (a) Dielectric loss $\varepsilon^{\prime \prime}$ and (b) dielectric loss modulus $\mathbf{M}^{\prime \prime}$ in the frequency domain for neat NR and its nanocomposites. Reproduced from ref. [7b] with permission from American Chemical Society. 


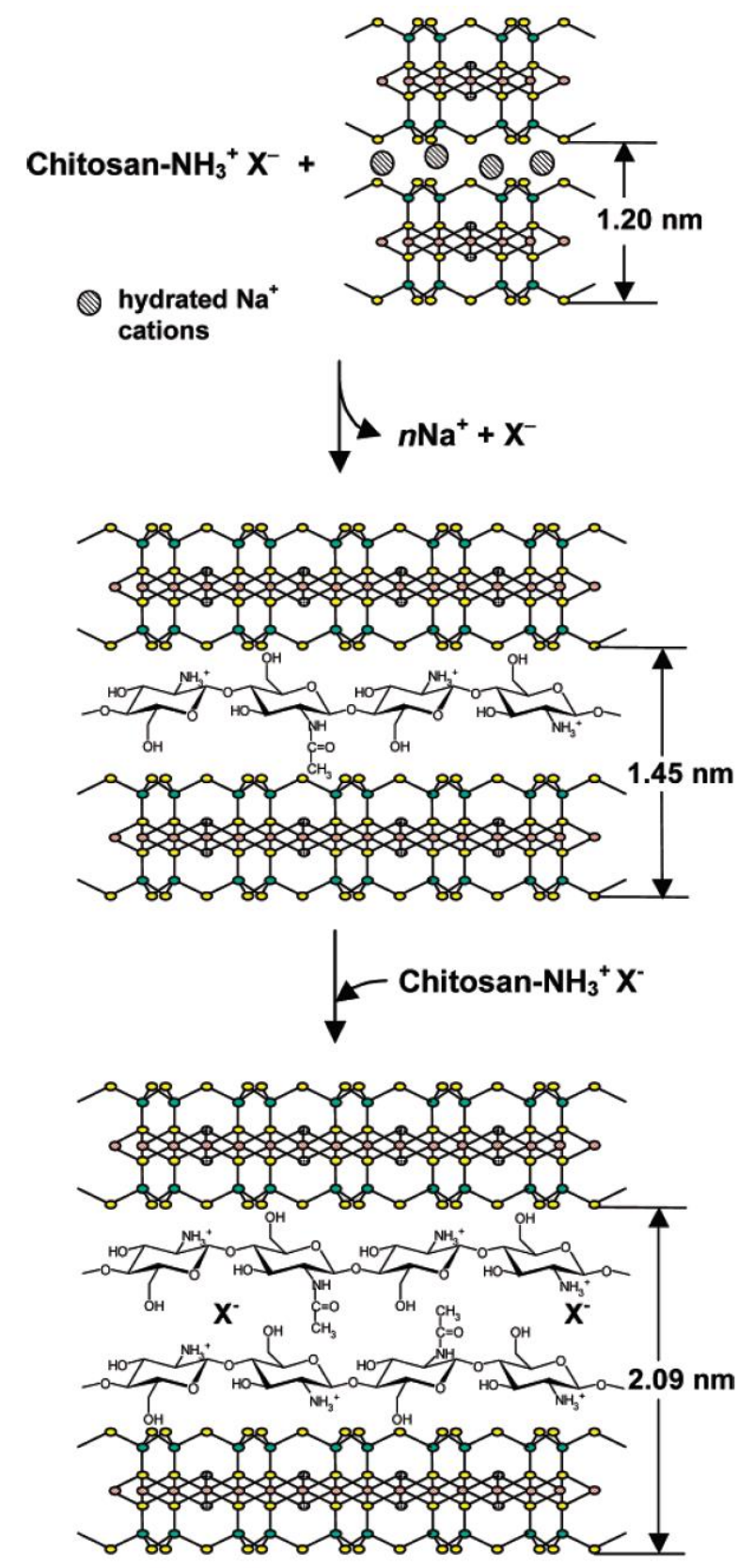

Figure 3. Scheme of the intercalation of Chitosan into Na+-Montmorillonite. Reproduced from ref. [16a] with permission from American Chemical Society. 